\title{
REVIEW ARTICLE \\ Systematic review of brucellosis in the Middle East: disease frequency in ruminants and humans and risk factors for human infection
}

\author{
I. I. MUSALLAM ${ }^{1 *}$, M. N. ABO-SHEHADA ${ }^{2}, Y$. M. HEGAZY ${ }^{3}, H \cdot R \cdot$ HOLT $^{1}$ AND \\ F. J. GUITIAN ${ }^{1}$ \\ ${ }^{1}$ Veterinary Epidemiology, Economics and Public Health Group, Department of Production and Population \\ Health, The Royal Veterinary College, University of London, Hatfield, UK \\ ${ }^{2}$ Faculty of Epidemiology and Population Health, London School of Hygiene and Tropical Medicine, Keppel \\ Street, London, UK \\ ${ }^{3}$ Department of Animal Medicine, Faculty of Veterinary Medicine, Kafr el-Sheikh University, Kafr el-Sheikh, Egypt
}

Received 4 January 2015; Final revision 14 September 2015; Accepted 1 October 2015;

first published online 28 October 2015

\section{SUMMARY}

A systematic review of studies providing frequency estimates of brucellosis in humans and ruminants and risk factors for Brucella spp. seropositivity in humans in the Middle East was conducted to collate current knowledge of brucellosis in this region. Eight databases were searched for peer-reviewed original Arabic, English, French and Persian journal articles; the search was conducted on June 2014. Two reviewers evaluated articles for inclusion based on predefined criteria. Of 451 research articles, only 87 articles passed the screening process and provided bacteriological and serological evidence for brucellosis in all Middle Eastern countries. Brucella melitensis and B. abortus have been identified in most countries in the Middle East, supporting the notion of widespread presence of Brucella spp. especially B. melitensis across the region. Of the 87 articles, 49 were used to provide evidence of the presence of Brucella spp. but only 11 provided new knowledge on the frequency of brucellosis in humans and ruminants or on human risk factors for seropositivity and were deemed of sufficient quality. Small ruminant populations in the region show seroprevalence values that are among the highest worldwide. Human cases are likely to arise from subpopulations occupationally exposed to ruminants or from the consumption of unpasteurized dairy products. The Middle East is in need of welldesigned observational studies that could generate reliable frequency estimates needed to assess the burden of disease and to inform disease control policies.

Key words: Brucella melitensis, B. abortus, human incidence, Middle East, ruminant prevalence, seroprevalence, systematic review.

\section{INTRODUCTION}

Brucellosis is a zoonotic disease that affects a wide range of animals including domestic livestock. It is

\footnotetext{
* Author for correspondence: Dr I. I. Musallam, Royal Veterinary College, Hawkshead Lane, North Mymms, Herts AL9 7TA, UK. (Email: Imusallam@rvc.ac.uk)
}

caused by members of the genus Brucella; among which; B. melitensis, B. abortus, B. suis, B. canis and $B$. ceti have been isolated from human cases in addition to their specific animal hosts [1-4]. Although accurate estimates of human incidence are lacking, largely because of under-reporting and misdiagnosis [5], brucellosis is considered one of the most common bacterial zoonoses worldwide [6]. 
In endemic areas, brucellosis is responsible for significant economic losses to livestock production due to abortions, reduced milk yield and infertility in addition to the public health burden [7]. The disease is transmitted to humans via the consumption of unpasteurized milk and dairy products from infected animals and through direct contact with afterbirth and aborted materials. As a result, individuals with occupational livestock contact in endemic areas, including farmers, abattoir workers, shepherds and veterinarians are at high risk $[8,9]$. The symptoms of human infection are non-specific, but the majority of patients with the acute form, present with fever, malaise, anorexia, headache, arthralgia, and backache. Persistent and recurrent fever is the most common clinical symptom in sub-acute cases. A small proportion of cases may develop complications including arthritis, endocarditis, spondylitis, sacroiliitis, osteomyelitis and meningoencephalitis $[9,10]$.

Infected livestock are the source of most human cases; therefore, prevention of human brucellosis is dependent on the control of the disease in livestock. This has been achieved with varying degrees of success using a combination of vaccination, test and slaughter of positive animals and quarantine/animal movement controls [11]. Cattle brucellosis, caused primarily by B. abortus, has been successfully eliminated from several countries including Japan, Canada, some European countries, Australia and New Zealand [12]. However, the control of B. melitensis in small ruminants appears to be more challenging than that of B. abortus, potentially as a result of its higher infectivity [13] as well as the characteristics of the livestock systems where it is endemic including increased mobility of small ruminant populations compared to large ruminants [7, 12]. Different control strategies have been recommended by the Food and Agriculture Organization (FAO) depending on the flock-/herd-level seroprevalence, therefore reliable disease frequency estimates are of great importance to inform and monitor the control programme. In lowprevalence areas $(<2 \%)$ test and slaughter of positive animals accompanied by sanitary measures is recommended. In settings where prevalence ranges between $2 \%$ and $10 \%$ the FAO advocates vaccination of young animals, non-compulsory vaccination of adult animals and test and slaughter of infected animals. In regions where prevalence is higher than $10 \%$, mass vaccination of all livestock is proposed as the optimal control strategy until a significant prevalence reduction is achieved and the strategy can be revised [11]. The appropriate strategy also depends on the socioeconomic context, the applied surveillance system, the policy set by the competent authorities as well as the baseline level of infection [14]. Ultimately, decisions on whether to prioritize brucellosis control over other diseases should ideally be informed by estimates of the human health burden expressed as disability adjusted life years (DALYs) and measures of monetary impact, i.e. economic losses due to human illness and decreased livestock productivity [7, 15]. The assumed high burden of the disease, particularly in low-income countries, is not matched by the attention it receives from health systems worldwide and as a result brucellosis has been included in the WHO's list of Neglected Zoonotic Diseases [6] Brucellosis is a major public health problem in the Middle East, Mediterranean region, and parts of Asia, Africa and Latin America [12, 16, 17]. In this paper, we focus on the Middle East, a region where brucellosis is assumed to be among the zoonoses with highest burden [18]. The region includes 15 countries; Bahrain, Egypt, Iran, Iraq, Israel, Jordan, Kuwait, Lebanon, Oman, Palestine, Qatar, Saudi Arabia, Syria, United Arab Emirates, and Yemen [19] (Fig. 1). Most of these countries have many similarities regarding livestock management systems, environmental conditions and culture [18]. Brucellosis is receiving increasing attention in the Middle East; some countries such as Egypt and Oman are implementing vaccination programmes for small and large ruminants whereas others, e.g. Iran, Iraq and Israel are adopting mass vaccination of small ruminants (Fig. 1). The aim of this study was to systemically identify, evaluate and summarize relevant published data on the presence and frequency of ruminant and human brucellosis in the Middle Eastern countries as well as on the strength of association between potential risk factors and Brucella. spp. seropositivity in humans.

\section{METHODS}

\section{Systematic review protocol}

A systematic review was conducted using a predefined protocol based on Cochrane [20] and PRISMA [21] guidelines. The protocol includes four main steps: (i) literature search to identify potential articles of relevance, (ii) screening for relevance, (iii) quality assessment and (iv) data extraction. Figure 2 summarizes the steps of the protocol with the number of papers that fulfilled the necessary criteria at each step.

\section{Search strategy and identification}

Eight electronic databases; BioMed Central Journals, CAB Direct (CABI), Cochrane Library-Cochrane 


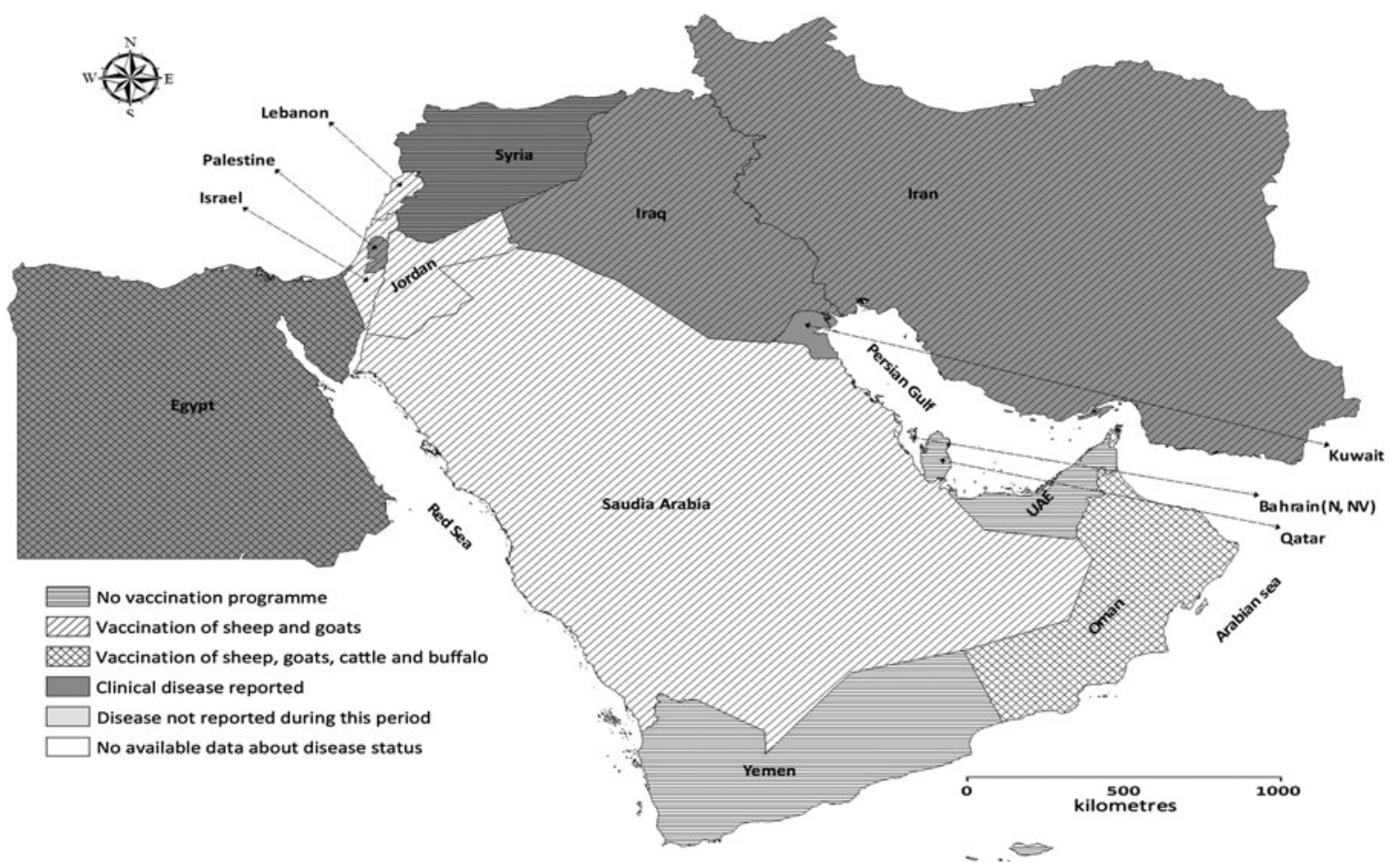

Fig. 1. Map of the Middle East showing ruminant brucellosis infection and vaccination status in different countries of the region, data obtained from OIE, 2013. N, Disease not reported; NV, no vaccination programme.

Database of Systematic Reviews (Wiley), Science Direct, ERIC plus Text (ProQuest), IBSS (CSA), PubMed, and Web of Science (ISI) Zetoc, were searched using the following terms:

(1) Brucellosis OR Malta fever OR Brucella OR 'Brucella melitensis' OR 'Brucella abortus'.

\section{AND}

(2) Middle East OR (Bahrain, Egypt, Iran, Iraq, Israel, Jordan, Kuwait, Lebanon, Oman, Palestine, Qatar, Saudi Arabia, Syria, United Arab Emirates, Yemen).

AND

(3) Humans OR Domestic AND Ruminants OR (Cattle OR Cow OR Bovine) OR (Sheep OR Ovine) OR (Goat OR Caprine) OR (Camel OR Camelidae OR Dromedary).

(4) Prevalence OR Incidence OR Risk AND Factors.

The search was conducted on June 2014 No time limits were set.

\section{Screening process}

All references were imported to EndNote (Thomson Reuters) and duplicated articles were excluded. The retrieved abstracts were screened by the primary author for entry into the next stage (quality assessment) based on the following inclusion criteria:

(1) The reported research is original and studies a human or animal population in one or more of the Middle Eastern countries.

AND

(2) The article is published in a peer-reviewed section of a journal.

\section{AND}

(3) The article is written in Arabic, English, French or Persian languages.

AND

(4) The research provides:

(a) Estimates of the frequency [(sero)-prevalence and/or incidence] of Brucella spp. infection in domestic ruminants and/or humans.

OR

(b) Estimates of the strength of association between Brucella spp. (sero)-positivity in humans and potential risk factors.

For articles that met the primary inclusion criteria or articles where the relevance could not be determined by reading the abstract alone; full texts were retrieved and the article was subjected to quality assessment and data extraction. 


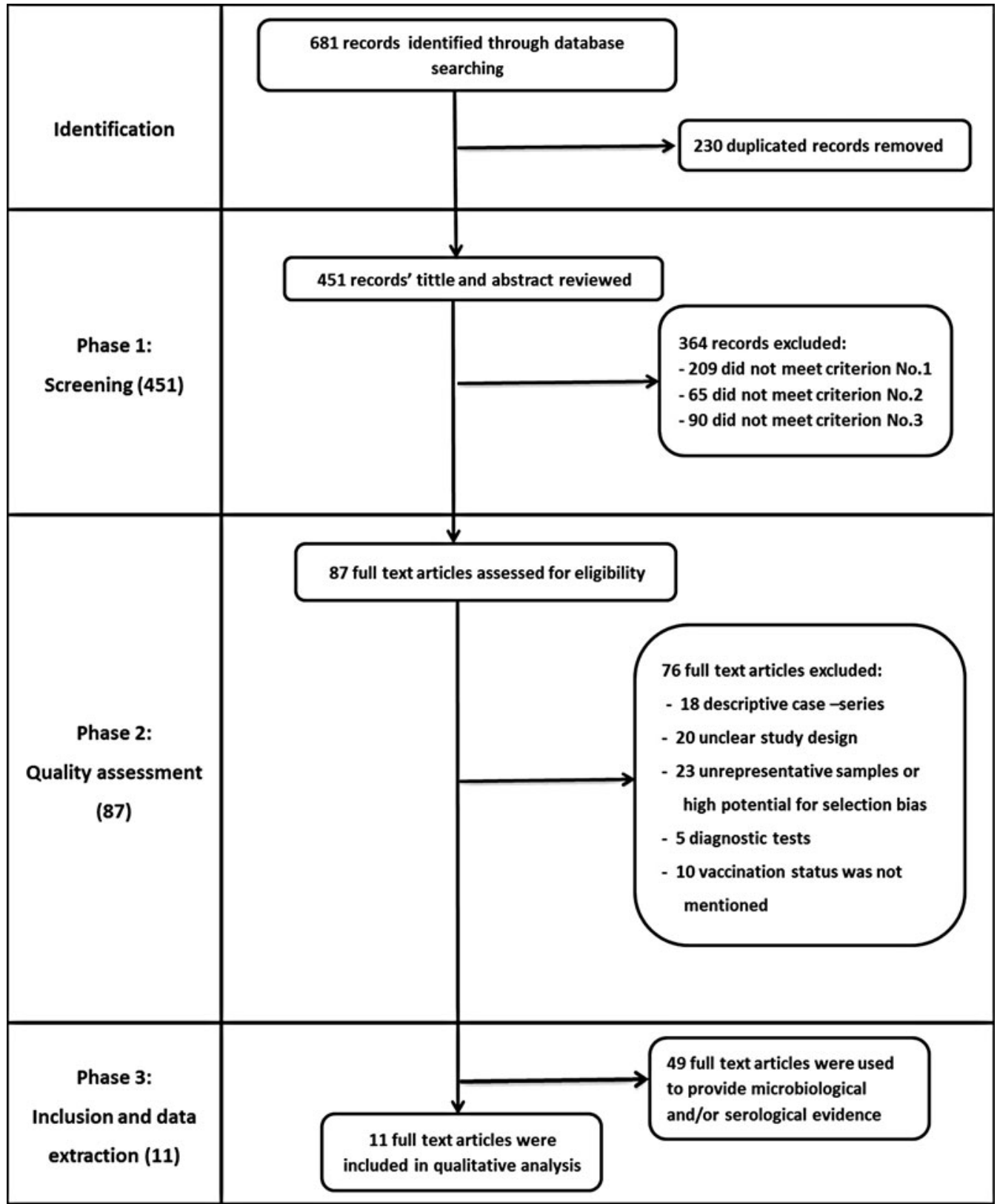

Fig. 2. Flow chart (template provided by PRISMA) showing the numbers of journal papers at each stage of the systematic review.

\section{Quality assessment and data extraction}

Two reviewers independently assessed the quality of the articles based on a set of criteria adapted from Cochrane guidelines [20], and Downs \& Black guidelines for cross-sectional studies [22]. Reviewers were first asked to specify the type of study, whether it was descriptive or analytical (i.e. involving an element of comparison across groups). Studies that were limited to the description of the characteristics of a series of cases (case reports or case series) were excluded. Reviewers were asked a series of questions to summarize the objectives, study design, study and target population, sampling strategy, diagnostic tests performed, statistical methods used and main outcomes of the study. These general questions were followed by a series of questions specific for each study type and to which reviewers could answer 'yes', 'no' or 'unclear'.

Selected studies were appraised by the two reviewers against the following five criteria and rated as 'high quality' studies when all five criteria were met:

(1) The type of study design was clear from the information provided. 
(2) Sampling strategy was clearly described, study unit was clearly stated (e.g. herd/flock $v s$. individual animal) with consideration of clustering if individual animals were targeted and the study population was considered fairly representative of the target population.

(3) The study was not deemed to have high potential for selection bias.

(4) Diagnostic tests used were those recommended by the WHO for humans and the World Organisation for Animal Health (OIE) for the studied species [23, 24]; given that no time limit was set in the search process, OIE and WHO recommendations at the time when the study was conducted were considered.

(5) The vaccination status of the study population was stated.

When provided, estimates of the frequency of seropositivity (incidence or true prevalence after adjusting for the imperfect performance of the used diagnostic tests) and of the strength of association (relative risks or odds ratios) where extracted.

Each reviewer extracted data independently using a data extraction form prepared by the primary author. Disagreements between reviewers were discussed in detail between them and resolved by consensus. The quality assessment checklist and data extraction forms are available upon request from the primary author.

\section{Data management}

Studies considered to be of 'high quality' were grouped according to whether they investigated; the frequency of brucellosis in humans, ruminants or risk factors for human seropositivity. Because of the heterogeneity within each group of studies in terms of study design, geographical areas, human or ruminant subpopulations under study and sample sizes, no statistical tests for heterogeneity or quantitative meta-analysis were performed; instead, data were extracted, summarized and organized in a qualitative manner.

Studies that passed the initial screening but did not fulfil the quality assessment criteria and were therefore deemed not to be of sufficient quality to generate unbiased estimates of frequency of disease or strength of association for human seropositivity were used to summarize available evidence of the presence of Brucella spp. in different host populations in the Middle East, where appropriate.

\section{RESULTS}

\section{Searching}

The initial search revealed 681 research articles, after removing duplicates 451 research articles remained, among these 23, 405, 3 and 20 were written in Arabic, English, French and Persian languages, respectively. Abstract screening was then performed and articles were excluded when they reported studies that were not carried out in one or more of the Middle Eastern countries (95 articles excluded), if they were not original research articles (114 articles excluded), if they were published in non-peer-reviewed journals (65 articles excluded) and if they did not provide estimates of brucellosis frequency in humans or domestic ruminants or potential risk factors for human seropositivity ( 90 articles excluded). A total of 87 articles (5 Arabic, 77 English, 5 Persian) met the primary eligibility criteria.

\section{Quality assessment}

During the quality assessment 76 articles were excluded for not fulfilling all five quality criteria listed in the quality assessment and data extraction part of the methods. Eighteen of studies were excluded because they were descriptive case-series, 43 studies were excluded due to unclear study design or nonrepresentative sampling therefore deemed to have high potential for selection bias. In five studies, the diagnostic tests used were not those recommended by WHO/OIE in the study species. Furthermore, in 10 studies the authors did not mention clearly whether the sampled animals were vaccinated or not, which may lead to inaccurate seroprevalence estimates. Of these 76 articles, 49 were retained and used to provide evidence of the presence of Brucella spp. in different ruminant hosts in Middle Eastern countries (Table 1). The range of the years of publication was 1974-2014 with a median of 2005. At least one $B$. melitensis biovar $(1,2,3)$ was identified in each country and at least one $B$. abortus biovar $(1,2,3,9)$ was identified in nine of the 15 countries supporting the widespread presence of Brucella spp. especially $B$. melitensis across the region. Moreover, B. suis biovar 1 was isolation from cattle in Egypt [25]. Only 11 articles were considered of sufficient quality and were used to provide frequency estimates in humans and ruminants or information on risk factors for brucellosis in humans. Table 2 describes the features of the eleven included studies. The number of papers that 
Table 1. Microbiological and serological evidences of the presence of Brucella melitensis and B. abortus (+) in humans and ruminants in the Middle East identified in a systematic review of peer-reviewed journal articles published in Arabic, English, French or Persian (search conducted on June 2014)

\begin{tabular}{|c|c|c|c|c|c|c|c|c|c|c|}
\hline \multirow[b]{3}{*}{ Country } & \multirow[b]{3}{*}{ Species [reference] } & \multicolumn{8}{|c|}{ Microbiological evidence } & \multirow{3}{*}{$\begin{array}{l}\text { Serological } \\
\text { evidence }\end{array}$} \\
\hline & & \multicolumn{5}{|c|}{ B. abortus (biovar) } & \multicolumn{3}{|c|}{$\begin{array}{l}\text { B. melitensis } \\
\text { (biovar) }\end{array}$} & \\
\hline & & 1 & 2 & 3 & 7 & 9 & 1 & 2 & 3 & \\
\hline \multirow[t]{4}{*}{ Bahrain } & Humans [26] & & & & & & & & + & + \\
\hline & Large ruminants & & & & & & & & & \\
\hline & Small ruminants & & & & & & & & & + \\
\hline & Camels & & & & & & & & & \\
\hline \multirow[t]{4}{*}{ Egypt } & Humans $[25,27,28]$ & & & & & & & & + & + \\
\hline & Large ruminants $[25,27,28]$ & + & & + & & & & & + & + \\
\hline & Small ruminants $[25,28]$ & & & & & & + & & + & + \\
\hline & Camels $[29,30]$ & + & & & + & & & & + & + \\
\hline \multirow[t]{4}{*}{ Iran } & Humans $[18,31,32]$ & & & & & & + & & + & + \\
\hline & Large ruminants $[31,32]$ & + & + & + & & + & + & + & & + \\
\hline & Small ruminants $[31,32]$ & & & & & & + & + & + & + \\
\hline & Camels $[33,34]$ & & & & & & & & + & + \\
\hline \multirow[t]{4}{*}{ Iraq } & Humans $[18,35]$ & & & & & & & & + & + \\
\hline & Large ruminants $[35,36]$ & & + & + & & + & + & & + & + \\
\hline & Small ruminants $[18,35]$ & & & & & & + & & + & + \\
\hline & Camels [37] & & & & & & & & & + \\
\hline \multirow[t]{4}{*}{ Israel } & Humans [38-40] & + & & + & & & + & + & + & + \\
\hline & Large ruminants $[18,41]$ & + & & + & & & & & + & + \\
\hline & Small ruminants $[38,40,41]$ & & & & & & + & + & + & + \\
\hline & Camels[39] & & & & & & + & & & \\
\hline \multirow{4}{*}{ Jordan } & Humans [42] & & & & & & & & + & + \\
\hline & Large ruminants $[43,44]$ & & & & & & + & & + & + \\
\hline & Small ruminants $[43,45-47]$ & & & & & + & + & & + & + \\
\hline & Camels [48] & & & & & & & & + & + \\
\hline \multirow{4}{*}{ Kuwait } & Humans [49] & & & & & + & + & & & + \\
\hline & Large ruminants [18] & & & & & & + & & & + \\
\hline & Small ruminants [18] & & & & & & + & & & + \\
\hline & Camels [50] & + & & & & & & & & + \\
\hline \multirow[t]{4}{*}{ Lebanon } & Humans $[51,52]$ & & & & & & & + & & + \\
\hline & Large ruminants & & & & & & & & & \\
\hline & Small ruminants [18] & & & & & & & + & + & + \\
\hline & Camels & & & & & & & & & \\
\hline \multirow[t]{4}{*}{ Oman } & Humans [53] & & & & & & + & & & + \\
\hline & Large ruminants [18] & & & & & + & & & & + \\
\hline & Small ruminants [18] & & & & & & + & & & + \\
\hline & Camels $[18,53]$ & & & & & & + & & & + \\
\hline \multirow[t]{4}{*}{ Palestine } & Humans $[54,55]$ & & & & & & & & + & + \\
\hline & Large ruminants & & & & & & & & & \\
\hline & Small ruminants $[56,57]$ & & & & & & & & + & + \\
\hline & Camels & & & & & & & & & \\
\hline \multirow[t]{4}{*}{ Qatar } & Humans [58] & & & & & & & & + & + \\
\hline & Large ruminants & & & & & & & & & \\
\hline & Small ruminants & & & & & & & & & \\
\hline & Camels & & & & & & & & & \\
\hline Saudi & Humans [59] & & & & & & & & + & + \\
\hline \multirow[t]{3}{*}{ Arabia } & Large ruminants [18] & & & & & & & & & + \\
\hline & Small ruminants [18] & & & & & & & & + & + \\
\hline & Camels [60] & & & & & & & & + & + \\
\hline
\end{tabular}


Table 1 (cont.)

\begin{tabular}{|c|c|c|c|c|c|c|c|c|c|c|}
\hline \multirow[b]{3}{*}{ Country } & \multirow[b]{3}{*}{ Species [reference] } & \multicolumn{8}{|c|}{ Microbiological evidence } & \multirow{3}{*}{$\begin{array}{l}\text { Serologica } \\
\text { evidence }\end{array}$} \\
\hline & & \multicolumn{5}{|c|}{ B. abortus (biovar) } & \multicolumn{3}{|c|}{$\begin{array}{l}\text { B. melitensis } \\
\text { (biovar) }\end{array}$} & \\
\hline & & 1 & 2 & 3 & 7 & 9 & 1 & 2 & 3 & \\
\hline \multirow[t]{4}{*}{ Syria } & Humans [61] & & & & & & + & & + & + \\
\hline & Large ruminants [61] & & & & & + & & & & + \\
\hline & Small ruminants $[18,61]$ & & & & & & + & & + & + \\
\hline & Camels & & & & & & & & & + \\
\hline \multirow[t]{4}{*}{ UAE } & Humans [62] & & & & & & + & + & + & + \\
\hline & Large ruminants & & & & & & + & & & + \\
\hline & Small ruminants $[62,63]$ & & & & & & + & + & + & + \\
\hline & Camels $[63,64]$ & & & + & & & & & & + \\
\hline \multirow[t]{4}{*}{ Yemen } & Humans [65] & & & & & & & & + & + \\
\hline & Large ruminants [66] & & & & & & & & & + \\
\hline & Small ruminants $[66,67]$ & & & & & & & & + & + \\
\hline & Camels [66] & & & & & & & & + & + \\
\hline
\end{tabular}

Table 2. Summary of studies on human and ruminant brucellosis in the Middle East deemed as relevant and of sufficient quality to be included in this systematic review describing the country, year, and type of study, diagnostic tests used and the main outcomes obtained

\begin{tabular}{|c|c|c|c|c|c|}
\hline Country [ref.] & Year & Type & Species studied & Diagnostic tests used & Main outcome \\
\hline Egypt [5] & 2002,2003 & $\begin{array}{l}\text { Population-based } \\
\text { surveillance }\end{array}$ & Human & STA & Annual incidence \\
\hline Saudi Arabia [68] & 1988 & Case-control & Human & STA, Coombs & Risk factors, OR \\
\hline Yemen [69] & 1991-1993 & Case-control & Human & STA & Risk factors, OR \\
\hline Iran [70] & 2005 & Case-control & Human & STA & Risk factors, OR \\
\hline Egypt [71] & 2003 & Case-control & Human & RBPT, TAT & Risk factors, OR \\
\hline Egypt [72] & 2007 & Case-control & Human & STA & Risk factors, OR \\
\hline Jordan [73] & 2013 & Case-control & Human & RBPT, ELISA & Risk factors, OR \\
\hline Jordan [45] & 2000-2001 & Cross-sectional & Sheep & RBPT, ELISA & $\mathrm{TP}$ \\
\hline Egypt [74] & 2008 & Cross-sectional & Cattle, buffalo, sheep, goats & RBPT, CFT, iELISA & $\mathrm{TP}$ \\
\hline Egypt [75] & 2005-2008 & Cross-sectional & Cattle, buffalo, sheep, goats & RBPT, CFT & $\mathrm{TP}$ \\
\hline Egypt [75] & 2009-2010 & Cross-sectional & Cattle, buffalo & iELISA & $\mathrm{TP}$ \\
\hline
\end{tabular}

CFT, Complement fixation test; ELISA, enzyme linked immnunosorbent assay; iELISA, indirect ELISA; OR, Odds ratio; RBPT, Rose Bengal precipitation test; STA, standard tube agglutination; TAT, tube agglutination test; TP, true seroprevalence.

passed the quality assessment step by country is presented in Figure 3.

\section{Frequency of brucellosis in humans}

The frequency of brucellosis in humans was investigated in many of the Middle Eastern countries but only one study [5] fully met the quality criteria. The study described a population-based surveillance for patients with acute febrile illness in an Egyptian governorate and estimated an annual incidence of brucellosis at 64 and 70/100 000 population in 2002 and 2003, respectively.

\section{Seroprevalence of brucellosis in ruminants}

A considerable number of studies assessed the frequency of brucellosis in different ruminant sub-populations in the Middle East. Four studies met the inclusion criteria; three in Egypt and one in Jordan. Table 3 summarizes the findings of these studies. Sheep, goat, cattle and 


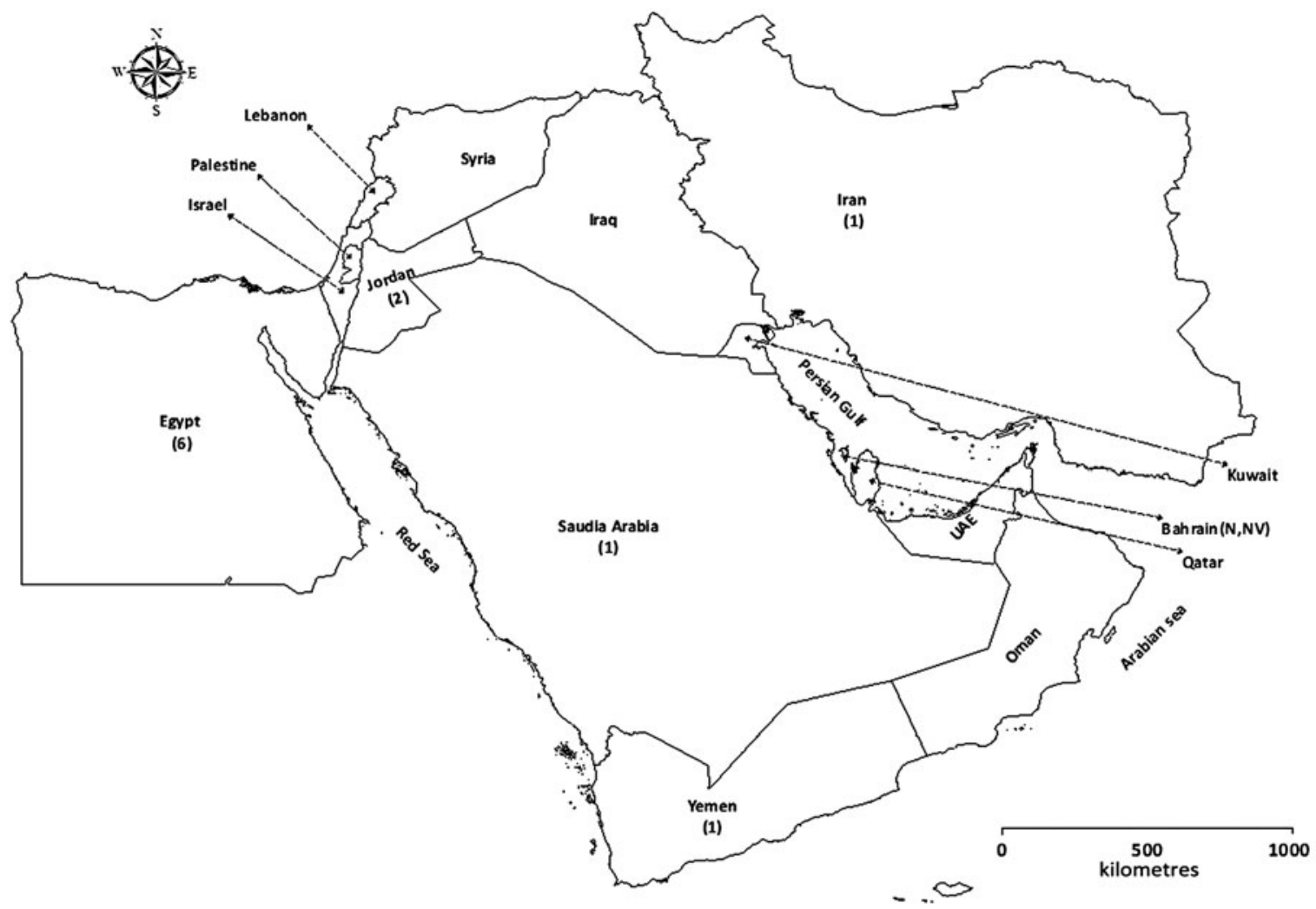

Fig. 3. Map of the Middle East showing countries with the number of studies per country deemed relevant and of sufficient quality to be included in this review in parentheses.

buffaloes were the studied species, and in all cases, frequency of infection was estimated as seroprevalence.

In Jordan, seroprevalence in Awassi sheep in the northern part of the country was estimated at $2 \cdot 2 \%$ [95\% confidence interval (CI) $0 \cdot 5-3 \cdot 5]$ and $45 \%(95 \%$ CI 32-58) at individual animal and flock levels, respectively, in 2000/2001 [45]. In Egypt, seroprevalence estimates for different livestock species are available for the Upper Egypt region and for the Kafr el-Sheikh Governorate (the largest governorate of the Nile Delta region). In Upper Egypt, true seroprevalence, after adjusting for imperfect test sensitivity and specificity, was estimated to be $1 \cdot 16$ (95\% CI $1 \cdot 05$ $1.27)$ in sheep, $0.44(95 \%$ CI $0.34-0.54)$ in goats, 0.79 $(95 \%$ CI $0 \cdot 71-0 \cdot 87)$ in cows and $0 \cdot 13(95 \%$ CI $0 \cdot 08-$ $0 \cdot 18)$ in buffaloes. These estimates were obtained from a study using secondary data for the period 2005-2008 in seven governorates of Upper Egypt [74]. In the Kafr el-Sheikh Governorate, a study conducted in 2008 [75] estimated true prevalence to be $12.2 \%$ (95\% CI 8.4-16.0) in individual sheep, $11 \cdot 3 \%(95 \%$ CI $7 \cdot 8-14 \cdot 8)$ in individual goats, $41 \cdot 3 \%(95 \%$ CI $26 \cdot 1-56 \cdot 7)$ in 'village flocks', $12 \cdot 2 \%(95 \%$ CI $7 \cdot 0-13 \cdot 3)$ in milk tanks from cows and $11 \cdot 3 \%$ (95\% CI 7.8-14.8) in milk tanks from buffaloes. A small study conducted in one single village in another governorate of the Nile Delta (Menufiya) estimated that $11 \%(95 \%$ CI 3.06-18.4) of unvaccinated individual cows and buffaloes had detectable antibodies in milk and that $15 \cdot 5 \%$ (95\% CI 6.61-24.7) of households keeping cows or buffaloes had at least one positive animal [76].

\section{Risk factors associated with human brucellosis in the Middle East}

The review identified six studies that measured the strength of association between potential risk factors and human brucellosis in the Middle East. All of them were case-control studies and were conducted in Iran, Saudi Arabia, Yemen, Jordan and Egypt (two studies). Details of these studies are summarized in Table 4 and the studied risk factors are summarized below.

\section{Consumption of dairy products}

Generally, the consumption of unpasteurized dairy products was a statistically significant risk factor for seropositivity in the Middle East. In Iran [70] and Jordan [73] sheep-derived, products were posing the 
Table 3. Summary of studies investigating the seroprevalence of brucellosis in different ruminant sub-populations in the Middle East deemed as relevant and of sufficient quality to be included in this systematic review

\begin{tabular}{lllll}
\hline \hline & & & \multicolumn{2}{l}{ True seroprevalence, \% $(95 \%$ CI) } \\
\cline { 4 - 5 } Country [ref.] & Level of study & Species & Individual level & Herd/flock level \\
\hline Jordan [45] & Sub-national & Sheep & $2 \cdot 2(0 \cdot 5-3 \cdot 5)$ & $56(44-69)$ \\
Egypt [74] & Governorate & Cattle & $0 \cdot 79(0 \cdot 71-0 \cdot 87)$ & $0 \cdot 2(0 \cdot 16-0 \cdot 23)$ \\
& & Buffalo & $0 \cdot 13(0 \cdot 08-0 \cdot 18)$ & \\
& & Sheep & $1 \cdot 16(1 \cdot 05-1 \cdot 27)$ & \\
Egypt [75] & Governorates & Cattle & $0 \cdot 44(0 \cdot 34-0 \cdot 54)$ & \\
& & Buffalo & $12 \cdot 2(7 \cdot 0-13 \cdot 3)$ & $15 \cdot 1(4 \cdot 0-26 \cdot 2)$ \\
& & Sheep & $12 \cdot 0(7 \cdot 1-13 \cdot 0)$ & $15 \cdot 1(4 \cdot 0-26 \cdot 2)$ \\
& & Goats & $12 \cdot 2(8 \cdot 4-16 \cdot 0)$ & $41 \cdot 3(26 \cdot 1-56 \cdot 7)$ \\
Egypt [76] & Village & Cattle, buffalo & $11 \cdot 3(7 \cdot 8-14 \cdot 8)$ & $32 \cdot 2(17 \cdot 8-46 \cdot 7)$ \\
\hline \hline
\end{tabular}

CI, Confidence interval.

Table 4. Risk factors for human brucellosis: summary of case-control studies deemed as relevant and of sufficient quality to be included in this systematic review

\begin{tabular}{|c|c|}
\hline Country [ref.], study population & Risk factors (OR, 95\% CI) \\
\hline $\begin{array}{l}\text { Saudi Arabia [68], } 150 \text { cases } \\
\text { and } 150 \text { controls }\end{array}$ & 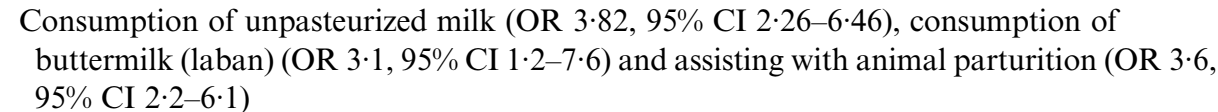 \\
\hline $\begin{array}{l}\text { Yemen [69], } 235 \text { cases and } 234 \\
\text { controls }\end{array}$ & 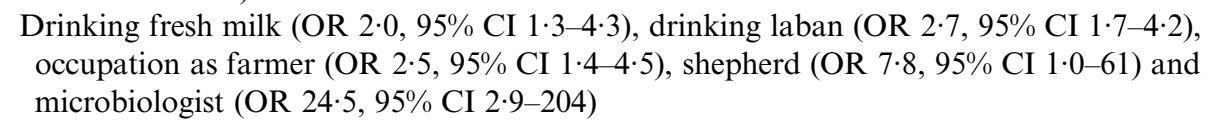 \\
\hline $\begin{array}{l}\text { Iran [70], } 150 \text { cases and } 150 \\
\text { matched controls }\end{array}$ & $\begin{array}{l}\text { Existence of another case of brucellosis in the home (OR 7.5, 95\% CI 3.9-14.6) and } \\
\text { consumption of unpasteurized dairy products (OR } 3 \cdot 7,95 \% \text { CI } 1 \cdot 6-8 \cdot 3)\end{array}$ \\
\hline $\begin{array}{l}\text { Egypt [71], } 149 \text { cases and } 298 \\
\text { controls }\end{array}$ & 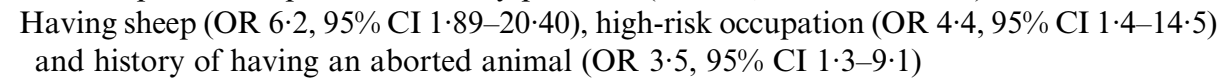 \\
\hline $\begin{array}{l}\text { Egypt [72], } 72 \text { cases and } 144 \\
\text { age-matched controls }\end{array}$ & $\begin{array}{l}\text { Direct contact with goats (OR } 3 \cdot 2,95 \% \text { CI } 1 \cdot 2-8 \cdot 7) \text {, occupations dealing with animals (OR } \\
2 \cdot 4,95 \% \text { CI } 1 \cdot 2-4 \cdot 9 \text { ) and eating ice cream from street vendors (OR } 2 \cdot 4,95 \% \text { CI } 1 \cdot 2-4 \cdot 6 \text { ) }\end{array}$ \\
\hline $\begin{array}{l}\text { Jordan [73], } 56 \text { cases and } 247 \\
\text { matched controls. }\end{array}$ & $\begin{array}{l}\text { Milking small ruminants (OR } 3 \cdot 5,95 \% \text { CI } 1 \cdot 5-8 \cdot 4) \text {, consumption of raw feta cheese (OR } 2 \cdot 8 \text {, } \\
95 \% \text { CI } 1 \cdot 4-5 \cdot 6 \text { ), consumption of cows' milk (OR } 0 \cdot 4,95 \% \text { CI } 0 \cdot 2-0 \cdot 8) \text { and the } \\
\text { consumption of boiled feta cheese (OR } 0 \cdot 4,95 \% \text { CI } 0 \cdot 2-0 \cdot 8)\end{array}$ \\
\hline
\end{tabular}

OR, Odds ratio; CI, confidence interval.

greatest risk. The study in Saudi Arabia [68] revealed that consumption of unpasteurized sheep and goat milk [odds ratio (OR) $3 \cdot 8,95 \%$ CI 2.2-6.4] and buttermilk (dairy product locally known as laban) (OR 3.0, $95 \%$ CI 1.2-7.6) were significant risk factors for seropositivity. In Egypt eating ice cream from street vendors increased the risk of seropositive status (OR $2 \cdot 4,95 \%$ CI 1·2-4·6) [71]. In Jordan, the consumption of raw feta cheese (OR 2.8, 95\% CI 1·4-5.6) was significantly associated with brucellosis, while the consumption of pasteurized cows' milk (OR 0.4, 95\% CI $0 \cdot 2-0 \cdot 8)$ and the consumption of boiled feta cheese
(OR $0 \cdot 4,95 \%$ CI $0 \cdot 2-0 \cdot 8$ ) decreased the risk of brucellosis [73].

\section{Occupational exposure}

Among exposures not associated with the foodborne route; contact with livestock, especially small ruminants, was a significant risk factor for Brucella spp. seropositivity in humans. Contact with sheep in Egypt (OR 6.2, 95\% CI 1.9-20.4) and direct contact with goats in Alexandria, Egypt (OR 3.2, 95\% CI $1 \cdot 2-8 \cdot 7)$ increased the risk of brucellosis [72]. 
Similarly, history of having an aborted animal (OR $3 \cdot 5,95 \%$ CI 1·3-9.1) in Egypt [71], assisting with animal parturition (in Jordan) (OR 3.6, CI 2.1-6.1) and milking small ruminants (in Jordan) were significant risk factors for seropositivity (OR 3.5, 95\% CI $1 \cdot 5$ 8.4) in humans [73].

Moreover, occupational contact with livestock and biological samples increased the risk of infection. Farmers (OR 2.5, 95\% CI 1.4-4.5), shepherds (OR $7 \cdot 8,95 \%$ CI 1.0-61) and microbiologists (OR 24.5, 95\% CI 2.9-204) were the groups at highest occupational risk of acquiring brucellosis in Yemen [69], and occupations dealing with animals in Alexandria, Egypt (OR 2.4, 95\% CI 1.2-4.9) had significantly increased risk [72].

Finally, the existence of another case of brucellosis in the home in Iran (OR 7.55, 95\% CI 3.9-14.6) was a major risk factor for seropositivity [70].

\section{DISCUSSION}

Brucellosis is considered endemic in most Middle Eastern countries where it is assumed to impose a considerable burden in terms of human disease and impaired livestock productivity [7, 10]. Our work aimed to systematically review available data regarding Brucella spp. presence and frequency estimates in humans and ruminants and associations between potential risk factors and human seropositive status in the Middle East. Although the primary search revealed 451 studies, after assessing their relevance, only 87 articles met the primary inclusion criteria and 49 of these provided evidence relevant for this review. Using strict quality criteria, only 11 studies were deemed of sufficient quality to provide reliable seroprevalence estimates that could eventually be used to quantify the burden of brucellosis in the region or data to inform disease prevention programmes prioritizing populations based on specific risk factors.

Most studies were excluded due to incomplete or unclear description of the design, or a design that was unlikely to generate unbiased estimates, including prevalence studies carried out using non-probabilistic sampling, studies comparing seroprevalence in purposively selected subpopulations without consideration of potential biases and studies where clustering of individual animals within herds was ignored. Most of the articles deemed of sufficient quality were produced in the last 15 years ( 9 of 11) and the other two articles were produced in the 1990s; moreover, 7 of 11 articles were collaborative work between
European or US and Middle Eastern researchers. This reflects the relatively modest and recent development of epidemiological research in the region and the importance of international collaboration.

Another critical issue with some studies was the use of diagnostic tests that are not recommended by the WHO/OIE for the host species being studied making the reliability of the obtained estimates questionable. Uncertainty with regard to the sensitivity and specificity of diagnostic tests being used hinders adjustment of observed apparent seroprevalence to obtain true seroprevalence estimates. Moreover, ignoring clustering of animals within the herd/flock during sampling will result in inaccurate estimates, given the use of imperfect diagnostics; herd specificity can be very low when several animals are tested in the same cluster and only one seropositive result is required for the herd to be classified as positive [77]. Diagnostic specificity may also be low due to cross-reactive bacteria or vaccination with smooth Brucella strains [78].

Lack of consideration of the vaccination status of the sampled animals was another reason why some studies were excluded, because vaccination of livestock against Brucella spp. is practiced in some Middle Eastern countries and the serological tests used were not able to differentiate between vaccinated and infected animals $[22,23]$ which can lead to overestimation of disease frequency. For example, two studies estimated the seroprevalence of brucellosis in sheep in Jordan; the first one was conducted in the northern governorates and reported seroprevalences of $2 \cdot 2 \%$ (95\% CI $0 \cdot 5-3 \cdot 5)$ and $56 \%(95 \%$ CI $44 \cdot 0-69 \cdot 0)$ at individual animal and flock levels, respectively [45]. The second was conducted in the southern governorates and reported seroprevalences of $37.6 \%$ and $47 \%$ (95\% CI 29-52) at individual animal and flock levels, respectively, the vaccination status of the sampled flocks was not mentioned in the second study. As mentioned previously, they may also have an issue of low herd specificity in these studies.

Although a considerable number of studies did not pass the quality assessment in this review, they provided evidence for Brucella seropositivity in all the countries of the region and all host species (Table 1) with a few exceptions: lack of evidence of seropositivity in large or small ruminants in Bahrain (where there is serological evidence in humans) and lack of evidence of seropositivity in large ruminants in Lebanon and Palestine (where there is evidence of seropositivity in small ruminants and humans). However, Lebanon, Palestine and Bahrain have 
small populations of ruminants compared to other countries in the region [18]. Further, the recent wars and relative instability in Lebanon and Gaza, and the restriction of movement in the Palestinian territories may be related to this. This may also be hindering collaborative work for the control of brucellosis between Middle Eastern countries and research work with international institutions. Data extracted from these studies show that B. melitensis biovars 1, 2, 3 and $B$. abortus biovars 1, 2, 3, 7 and 9 were the most frequently isolated Brucella spp. in the majority of the Middle Eastern countries and recently, molecular work in Egypt has shown the presence of B. suis biovar 1 in milk and lymph node samples from cattle [25]. Frequent isolation of B. melitensis from cattle in the Middle East raises questions on the role of cattle in disease maintenance and transmission, which needs further investigation. Moreover, it highlights the role of mixing small and large ruminants which is practices in most of the Middle East countries [18].

Despite the scarcity of sound prevalence or incidence estimates, the review found serological evidence of Brucella spp. in humans in all countries (Table 1). Isolation of $B$. abortus from humans only in Israel could be the result of transmission from cattle to humans before it has been eliminated from the country after adapting vaccination programme [38].

Based on our inclusion criteria, only one study provides good quality estimates of the frequency of brucellosis in humans. This was a population-based surveillance implemented in Fayoum Governorate in Egypt in 2002 and 2003 [5]. Most studies concerning human infection consisted of case-series describing cases retrospectively using data from hospital records without a control group, therefore precluding the investigation of risk factors for infection. Other studies have investigated the prevalence among high risk subpopulations such as nomadic people or among patients who suffered manifestations compatible with infection such as women with miscarriage. Such studies were excluded when selection of individuals was not done probabilistically, although the estimates provided by these studies could be of use and in fact, have been included in a recently published review on human brucellosis, commissioned by the WHO [79]; the reason for their inclusion was to fill gaps in some countries to offer frequency estimates to be used in the calculation of DALYs for human brucellosis.

Studies estimating the frequency of brucellosis in humans in the Middle East often rely on the use of records of public hospitals and primary health centres.
Such records depend largely on the clinical presentation of the disease rather than laboratory confirmation. Furthermore, a considerable number of cases do not seek medical care or may be referred to private health centres rather than official ones. As a result, such records would result in estimates that are unreliable. There is a need for population-based surveillance combining clinical presentations and laboratory confirmation [5].

Although the seroprevalence of brucellosis in ruminants has been intensively investigated across the Middle East, the current review identified only four studies of sufficient quality reporting seroprevalence in four ruminant sub-populations; sheep, goats, cattle and buffalos in two countries; Egypt and Jordan [45, 74-76].

The reported seroprevalence varied widely from country to country and even between regions within the same country. In Egypt, for example, the true seroprevalence at individual animal level in sheep was estimated at $1 \cdot 16(95 \%$ CI $1 \cdot 05-1 \cdot 27)$ in seven of Upper Egypt governorates, whereas it was estimated as $12.2 \%(95 \%$ CI $8 \cdot 4-16 \cdot 0)$ in one governorate of the Nile Delta. The results of Upper Egypt study [74] are similar to the results of the Jordanian study which reported a true seroprevalence of $2 \cdot 2 \%(95 \%$ CI $0 \cdot 5-3 \cdot 5)$ at individual animal level [45]. At flock or village levels the relatively high reported true seroprevalence values for sheep in Egypt at 41.3\% (95\% CI $26 \cdot 1-56 \cdot 7)$ and in Jordan at $45 \%(95 \%$ CI $32-58)$ were thought to be the result of free uncontrolled movement of sheep flocks between villages, which facilitates contact between infected and susceptible animals [45, 74] and has implications for the likely success of control programmes. This finding is of high importance and supports the notion that brucellosis is widespread, at least in some Middle Eastern countries, with flocklevel seroprevalence estimates which are among the highest when compared with endemic situations reported in other parts of the world. Moreover, animal movement between different countries in the region and the intense animal movement between the Horn of Africa and the Middle East for trading represent a challenge for the control and require more collaboration at the international level.

Available estimates suggest that brucellosis is endemic at high levels not only among small ruminants but also in bovine subpopulations in Egypt, and the reported seroprevalence in Egyptian cattle and buffalo herds varied between governorates [74-76]. This variation in the estimates could result from the 
heterogeneity of studied populations in terms of husbandry practices and livestock densities as well as different environmental conditions.

Risk factors for human seropositivity with Brucella spp. can be grouped into two main categories; direct contact with animals, particularly material from abortion or parturition, and consumption of contaminated milk and dairy products from infected animals. These high risk practices, coupled with lack of sufficient knowledge of the disease and absence of effective prevention strategies result in maintenance of the disease in the region [80]. Due to cultural and livestock management similarities in Middle Eastern countries it may be possible to extrapolate findings with regard to risk factors identified in one country to other countries. In Saudi Arabia and Yemen, consumption of raw milk and other dairy products appeared to be a greater risk factor for human seropositivity compared to direct contact with animals [68, 69]. Conversely, studies from Iran and Egypt identified direct contact with infected animals to be more significantly associated with seropositivity [70-72]. The reason for these differences in the identified risk factors can be attributed to the nature of the populations under study (e.g. urban $v s$. rural). The finding of microbiologists as a high-risk occupational group in a study in Yemen highlights the need of ensuring and promoting biosafety at the same time as diagnostic capacity. Brucellosis is one of the most common laboratory infections, particularly in developing countries, which may not have adequate regulations and enforcements regarding laboratory safety. Transmission can occur via sniffing plates, working with viable organisms outside the safety cabinet, not using protective equipment such as gloves and masks or ingesting suspensions of living organism during mouth pipetting. Developing standard procedures and training staff in good laboratory practices during handling of viable organisms or biological samples and the use of biosafety practices will help mitigate the risk of acquiring the infection [81].

Countries in the region are facing similar challenges: endemicity of one or more Brucella spp. in their ruminant populations, inconsistent vaccination practices and difficulties associated with the structure of the production systems and resources (e.g. lack of animal identification, precluding quarantine and movement control implementation). Furthermore, political turmoil, war, human displacement and competing needs for limited resources impede the implementation of national control programmes in some countries. As a result, much needed coordinated action against brucellosis in the region would be challenging.

Although the number of studies that fulfil the quality criteria of this review is small, data presented in these studies indicate that ruminant brucellosis is endemic at high levels in both small and large ruminants in some Middle Eastern countries, such as Egypt and Jordan. Sound epidemiological research is crucial to inform the design of realistic control programmes [82]. Unbiased estimates of frequency of infection are needed to assess whether elimination by means of test and slaughter is a realistic short-term objective or, conversely, large-scale vaccination is advisable to reduce the prevalence of infection to levels that make elimination more feasible. It has been shown that lack of a clear delineation between these two objectives (prevalence reduction $v s$. elimination) official control programmes has contributed to their erratic implementation in some of the Middle Eastern countries [83]. Furthermore, epidemiological evidence on the distribution of infection across geographical areas, production systems and affected ruminant species, ideally accompanied by the identification and characterization of circulating Brucella spp., can inform more targeted and effective surveillance and control efforts.

This review highlights the need for more detailed information on the frequency and distribution of infection and its associated burden to identify the most cost-effective options for control. However, based on available evidence it is likely that strategic vaccination of ruminant populations combined with sustained surveillance systems and public health education programmes may be the most appropriate control strategy. The lack of good quality estimates demonstrates the need for more comprehensive and welldesigned epidemiological studies to bridge the current gap in brucellosis knowledge in the Middle East; this can be achieved through regional and international collaboration. At the regional level, competent authorities should develop sustainable surveillance systems, apply strict monitoring programmes on livestock movement and provide training programmes for both; veterinarians and provincial doctors in the region.

At the international level, technical and financial support should be directed to endemic areas in the world such as the Middle East.

\section{CONCLUSIONS}

Brucellosis remains a major public health problem in the Middle East and available evidence, although 
limited, supports this belief. Cases are likely to arise from subpopulations directly exposed to ruminants or from the consumption of unpasteurized dairy products from infected ruminants, with some ruminant subpopulations in the region showing among the highest seroprevalence levels compared to other endemic regions. Serological and microbiological evidence supports the widespread presence of Brucella spp. across the region. However, there is a lack of reliable estimates of the frequency of disease both in humans and livestock which precludes the formulation of multi-sectorial control policies. There is a need for well-designed observational studies that could generate reliable frequency estimates needed to assess the burden of disease and to inform disease control policies.

\section{ACKNOWLEDGEMENTS}

This work was supported by Youssef Jameel Scholarship fund.

\section{DECLARATION OF INTEREST}

None.

\section{REFERENCES}

1. Muma JB, et al. Brucella seroprevalence of the Kafue lechwe (Kobus leche kafuensis) and black lechwe (Kobus leche smithemani): exposure associated to contact with cattle. Preventive Veterinary Medicine 2011; 100: 256-260.

2. Sohn AH, et al. Human neurobrucellosis with intracerebral granuloma caused by a marine mammal Brucella spp. Emerging Infectious Diseases 2003; 9: 485-488.

3. Foster G, et al. Brucella ceti sp. nov. and Brucella pinnipedialis sp. nov. for Brucella strains with cetaceans and seals as their preferred hosts. International Journal of Systematic and Evolutionary Microbiology 2007; 57: 2688-2693.

4. Nagalingam M, et al. Molecular typing of Brucella species isolates from livestock and human. Tropical Animal Health and Production 2012; 44: 5-9.

5. Jennings GJ, et al. Brucellosis as a cause of acute febrile illness in Egypt. Transactions of the Royal Society of Tropical Medicine and Hygiene 2007; 101: 707-713.

6. World Health Organization. Seven neglected endemic zoonoses - some basic facts. (http://www.who.int/zoonoses/neglectedzoonoticdiseases/en/), 2009. Accessed March 2014.

7. McDermott J, Grace D, Zinsstag J. Economics of brucellosis impact and control in low-income countries. Revue Scientifique et Technique (International Office of Epizootics) 2013; 32: 249-261.
8. Seleem MN, Boyle SM, Sriranganathan N. Brucellosis: a re-emerging zoonosis. Veterinary Microbiology 2010; 140: $392-398$.

9. Doganay M, Aygen B. Human brucellosis: an overview. International Journal of Infectious Diseases 2003; 7: 173-182.

10. Dean AS, et al. Clinical manifestations of human brucellosis: A systematic review and meta-analysis. PLOS Neglected Tropical Diseases 2012; 6: e1929.

11. Food and Agriculture Organization. Brucella melitensis in Eurasia and the Middle East. FAO Animal Production and Health Proceedings 2010. No. 10. Rome.

12. The Centre for Food Security and Public Health. Ovine and caprine brucellosis: Brucella melitensis. CFSPH, Iowa State University, Iowa, USA, 2009 (http://www. cfsph.iastate.edu/Factsheets/pdfs/brucellosismelitensis. pdf). Accessed March 2014.

13. Cloeckaert A, et al. Major outer membrane proteins of Brucella spp.: past, present and future. Veterinary Microbiology 2002; 90: 229-247.

14. Benkirane A. Epidemiologic surveillance and prevention of brucellosis in ruminants: the example of the North African region and the Near East. Revue Scientifique et Technique(International Office of Epizootics) 2001; 20: 757-767.

15. Murray CJ. Quantifying the burden of disease: the technical basis for disability-adjusted life years. Bulletin of the World Health Organization 1994; 72: 429.

16. Gwida M, et al. Brucellosis - regionally emerging zoonotic disease. Croatian Medical Journal 2010; 51: 289-295.

17. Pappas G, et al. The new global map of human brucellosis. Lancet Infectious Diseases 2006; 6: 91-99.

18. Refai M. Incidence and control of brucellosis in the Near East region. Veterinary Microbiology 2002; 90: 81-110.

19. World Bank. Middle East and North Africa regional brief 2011 (http://web.worldbank.org/WBSITE/ EXTERNAL/COUNTRIES/). Accessed March 2014.

20. Higgins JP, Green S. Cochrane Handbook for Systematic Reviews of Interventions. Wiley Online Library, 2008.

21. Moher D, et al. Preferred reporting items for systematic reviews and meta-analyses: the PRISMA statement. Annals of Internal Medicine 2009; 151: 264-269.

22. Downs SH, Black N. The feasibility of creating a checklist for the assessment of the methodological quality both of randomised and non-randomised studies of health care interventions. Journal of Epidemiology and Community Health 1998; 52: 377-384.

23. OIE. Manuals of Diagnostic Tests and Vaccines for Terrestrial Animals 2009, chapter 2.4.3 (Bovine brucellosis) (http://oie.int/fr/normes/manua/2009/pdf/2.04.03_ Bovine_brucella.pdf). Accessed March 2014.

24. OIE. Manuals of Diagnostic Tests and Vaccines for Terrestrial Animals 2009, chapter 2.7.7 (Caprine and Ovine brucellosis) (http://oie.int/fr/normes/manua/ 2009/pdf/2.07.02_caprine_ovine_brucella.pdf). Accessed March 2014.

25. Menshawy AM, et al. Assessment of genetic diversity of zoonotic Brucella spp. recovered from livestock in Egypt 
using multiple locus VNTR analysis. BioMed Research International 2014; 2014: 353876.

26. Ali R, et al. Brucellosis with spondylitis. Bahrain Medical Bulletin 2000; 7: 1-8.

27. Samaha H, et al. Serodiagnosis of brucellosis in cattle and humans in Egypt. Immunobiology 2009; 214: 223-226.

28. Hamdy ME, Amin AS. Detection of Brucella species in the milk of infected cattle, sheep, goats and camels by PCR. Veterinary Journal 2002; 163: 299-305.

29. Nada AR, et al. Studies on brucellosis in camels in Egypt. Egyptian Journal of Veterinary Sciences 1991; 28: $91-101$.

30. El-Seedy FR, Radwan AI, El-Shabrawy MA. Serological and bacteriological investigations on Brucella infection in one humped camels, Camelus dromedarius in Egypt. Veterinary Medical Journal of Cairo University. 2000; 48: 83-89.

31. Akbarmehr J, Ghiyamirad M. Serological survey of brucellosis in livestock animals in Sarab City (East Azarbayjan province), Iran. African Journal of Microbiology Research 2011; 5: 1220-1223.

32. Behroozikhah A, et al. Differentiation of Iranian strains of Brucella spp. by random amplification of polymorphic DNA. Archives of Razi Institue 2005; 59: 13-23.

33. Zowghi E, Ebadi A. Brucellosis in camels in Iran. Revue Scientifique et Technique (International Office of Epizootics) 1988; 7: 383-386.

34. Khadjeh G, Zowghi E, Zarif-Fard MR. Incidence of brucellosis in one-humped camels of Boushehr, Iran. Archives of Razi Institue 1999; 50: 83-86.

35. Abbas BA, Aldewan AB. Occurrence and epidemiology of Brucella spp in raw milk samples at Basrah province, Iraq. Bulgarian Journal of Veterinary Medicine 2009; 12: 136-142.

36. Shareef JM. A Review of serological investigations of brucellosis among farm animals and humans in northern provinces of Iraq (1974-2004). Journal of Veterinary Medicine, Series B 2006; 53: 38-40.

37. Yawoz M, et al. A serological study of brucellosis in camels south of Kirkuk, Iraq. Iraqi Journal of Veterinary Sciences 2012; 26: 105-107.

38. Shemesh AA, Yagupsky P. Increasing incidence of human brucellosis in southern Israel after the cessation of a veterinarian control campaign. Air \& Water Borne Diseases Journal 2013; 2: 10.4172.

39. Shimol SB, et al. Human brucellosis outbreak acquired through camel milk ingestion in southern Israel. The Israel Medical Association Journal 2012; 14: 475-478.

40. Banai M, Mayer I, Cohen A. Isolation, identification, and characterization in Israel of Brucella melitensis biovar 1 atypical strains susceptible to dyes and penicillin, indicating the evolution of a new variant. Journal of Clinical Microbiology 1990; 28: 1057-1059.

41. Bardenstein S, et al. Identification of the Brucella melitensis vaccine strain Rev.1 in animals and humans in Israel by PCR analysis of the PstI site polymorphism of its omp2 gene. Journal of Clinical Microbiology 2002; 40: 1475-1480.

42. Al-Majali AM, Shorman M. Childhood brucellosis in Jordan: prevalence and analysis of risk factors.
International Journal of Infectious Diseases 2009; 13: 196-200.

43. Aldomy FM, Jahans KL, Altarazi YH. Isolation of Brucella melitensis from aborting ruminants in Jordan. Journal of Comparative Pathology 1992; 107: 239-242.

44. Al-Majali AM, Talafha AQ, Ababneh MM. Seroprevalence and risk factors for bovine brucellosis in Jordan. Journal of Veterinary Sciences 2009; 10: 6165.

45. Al-Talafhah AH, Lafi SQ, Al-Tarazi Y. Epidemiology of ovine brucellosis in Awassi sheep in Northern Jordan. Preventive Veterinary Medicine 2003; 60: 297-306.

46. Al-Majali AM, et al. Prevalence of, and risk factors for, brucellosis in Awassi sheep in Southern Jordan. Small Ruminant Research 2007; 73: 300-303.

47. Al-Majali AM. Seroepidemiology of caprine brucellosis in Jordan. Small Ruminant Research 2005; 58: 13-18.

48. Dawood HA. Brucellosis in camels (Camelus dromedorius) in the south province of Jordan. American Journal of Agricultural and Biological Sciences 2008; 3: 623-626.

49. Al-Nakkas A, Mustafa AS, Wright SG. Large-scale evaluation of a single-tube nested PCR for the laboratory diagnosis of human brucellosis in Kuwait. Journal of Medical Microbiology 2005; 54: 727-730.

50. Al-Khalaf S, El-Khaladi A. Brucellosis of camels in Kuwait. Comparative Immunology, Microbiology \& Infectious Diseases 1989; 12: 1-4.

51. Araj G, Azzam R. Seroprevalence of Brucella antibodies among persons in high-risk occupation in Lebanon. Epidemiology and Infection 1996; 117: 281-288.

52. Kattar MM, et al. Evaluation of a multilocus variable-number tandem-repeat analysis scheme for typing human Brucella isolates in a region of brucellosis endemicity. Journal of Clinical Microbiology 2008; 46: 3935-3940.

53. El-Amin EO, et al. Brucellosis in children of Dhofar Region, Oman. Saudi Medical Journal 2001; 22: 610-615.

54. Husseini AS, Ramlawi AM. Brucellosis in the West Bank, Palestine. Saudi Medical Journal 2004; 25: 1640-1643.

55. Awad R. Human brucellosis in the Gaza Strip, Palestine. Eastern Mediterranean Health Journal 1998; 4: 225-233.

56. Awwad E, et al. Isolation, identification and molecular characterization of Brucella strains isolated from small ruminants in the West Bank, Palestine. Revue de Médecine Vétérinaire 2011; 162: 236-239.

57. Hawari AD. Epidemiological studies, seroprevalance and some risk factors of brucellosis in sheep and goats in the South Province of West Bank. Asian Journal of Animal and Veterinary Advances 2012; 7: 535-539.

58. Rahil AI, et al. Brucellosis in Qatar: a retrospective cohort study. Qatar Medical Journal 2014; 2014: 25-30.

59. Asaad AM, Alqahtani JM. Serological and molecular diagnosis of human brucellosis in Najran, Southwestern Saudi Arabia. Journal of Infection and Public Health 2012; 5: 189-194.

60. Radwan AI, Bekairi SI, Prasad PV. Serological and bacteriological study of brucellosis in camels in central Saudi Arabia. Revue Scientifique et Technique (International Office of Epizootics) 1992; 11: 837-844. 
61. Darwish M, Benkirane A. Field investigations of brucellosis in cattle and small ruminants in Syria, 1990-1996. Revue Scientifique et Technique (International Office of Epizootics) 2001; 20: 769-775.

62. Goodwin CS. First Report of a strain of Brucella melitensis that was widely sensitive to brucellaphages isolated in the United Arab Emirates. Clinical Infectious Diseases 1996; 22: 190-191.

63. Mohammed MA, Shigidy MT, Al-Juboori AY. Sero-prevalence and epidemiology of brucellosis in camels, sheep and goats in Abu Dhabi emirate. International Journal of Animal and Veterinary Advances 2013; 5: 82-86.

64. Moustafa T, et al. Surveillance of Brucella antibodies in camels of the eastern region of Abu Dhabi, United Arab Emirates. Proceedings of the Third Annual Meeting for Animal Production under Arid Conditions, 1998; 1: 160-166.

65. AL-Shamahy HA. The prevalence of Brucella antibodies in Yemen. Saudi Medical Journal 1997; 18: 45-48.

66. Al-Shamahy HA. Seropositivity for brucellosis in a sample of animals in the Republic of Yemen. Eastern Mediterranean Health Journal 1999; 5: 1042-1044.

67. Hosie B, Al-Bakri O, Futter R. Survey of brucellosis in goats and sheep in the Yemen Arab republic: Comparison of tests for Brucella melitensis infection in sheep. Tropical Animal Health and Production 1985; 17: 93-99.

68. Cooper CW. Risk factors in transmission of brucellosis from animals to humans in Saudi Arabia. Transactions of the Royal Society of Tropical Medicine and Hygiene 1992; 86: 206-209.

69. Al-Shamahy HA, Whitty CJ, Wright SG. Risk factors for human brucellosis in Yemen: a case control study. Epidemiology and Infection 2000; 125: 309-313.

70. Sofian M, et al. Risk factors for human brucellosis in Iran: a case-control study. International Journal of Infectious Diseases 2008; 12: 157-161.

71. El Sherbini A, et al. Risk factors and diagnostic criteria of brucellosis in an endemic area in Egypt. Infectious Diseases in Clinical Practice 2005; 13: 295-299.

72. Meky FA, et al. Epidemiology and risk factors of brucellosis in Alexandria governorate. Eastern Mediterranean Health Journal 2007; 13: 677-685.
73. Abo-Shehada MN, Abu-Halaweh M. Risk factors for human brucellosis in northern Jordan. Eastern Mediterranean Health Journal 2013; 19: 32-37.

74. Hegazy YM, et al. Ruminant brucellosis in Upper Egypt (2005-2008). Preventive Veterinary Medicine 2011; 101: 173-181.

75. Hegazy YM, et al. Ruminant brucellosis in the Kafr El Sheikh governorate of the Nile Delta, Egypt: prevalence of a neglected zoonosis. PLoS Neglected Tropical Diseases. 2011; 5: e944.

76. Holt HR, et al. Brucella spp. infection in large ruminants in an endemic area of Egypt: cross-sectional study investigating seroprevalence, risk factors and livestock owner's knowledge, attitudes and practices (KAPs). BMC Public Health 2011; 11: 341.

77. Dohoo IR, Martin W, Stryhn H. Veterinary Epidemiologic Research, 2nd edn. Canada: AVC Incorporated, 2010, pp. 125.

78. McGiven JA. New developments in the immunodiagnosis of brucellosis in livestock and wildlife. Revue Scientifique et technique (International Office of Epizootics) 2013; 32: 163-176.

79. Dean AS, et al. Global burden of human brucellosis: a systematic review of disease frequency. PLoS Neglected Tropical Diseases. 2012; 6: e1865.

80. Tebug SF. Factors associated with milk producer's awareness and practices in relation to zoonoses in northern Malawi. Veterinary World 2013; 6: 249-253.

81. Yagupsky P, Baron EJ. Laboratory exposures to brucellae and implications for bioterrorism. Emerging Infectious Diseases 2005; 11: 1180-1185.

82. Okello A, Welburn S, Smith J. Crossing institutional boundaries: mapping the policy process for improved control of endemic and neglected zoonoses in sub-Saharan Africa. Health policy and Planning. Published online: 7 July 2014. doi:10.1093/heapol/ czu059.

83. Hegazy YM, Ridler AL, Guitian FJ. Assessment and simulation of the implementation of brucellosis control programme in an endemic area of the Middle East. Epidemiology and Infection 2009; 137: 14361448. 